\title{
Elastic Theory Has Zero Radius of Convergence
}

\author{
Alex Buchel and James P. Sethna \\ Laboratory of Atomic and Solid State Physics, \\ Cornell University, Ithaca, NY, 14853-2501
}

\begin{abstract}
Nonlinear elastic theory studies the elastic constants of a material (such as Young's modulus or bulk modulus) as a power series in the applied load. The inverse bulk modulus $\mathrm{K}$, for example depends on the compression P: $1 / K(P)=c_{0}+c_{1} P+c_{2} P^{2} \cdots+c_{n} P^{n}+\cdots$. Elastic materials that allow cracks are unstable at finite temperature with respect to fracture under a stretching load; as a result, the above power series has zero radius of convergence and thus can at best be an asymptotic series. Considering thermal nucleation of cracks in a two-dimensional isotropic, linearelastic material at finite temperature we compute the asymptotic form $c_{n+1} / c_{n} \rightarrow C n^{1 / 2}$ as $n \rightarrow \infty$. We present an explicit formula for $C$ as a function of temperature and material properties.
\end{abstract}

PACS numbers: 46.30.Nz, 62.20.Mk, 64.60.Qb

Hooke's law, $F=k x$, representing the elastic response of a body to an external stress, is only the first term in a Taylor's series. This paper addresses the convergence properties of this expansion. We argue generally that the radius of convergence of elastic theory is zero, and for two-dimensional isotropic linear-elastic theory allowing for brittle fracture, we can calculate the asymptotic behavior of the coefficients.

For simplicity, let's consider the bulk modulus $K(P)$,

$$
\frac{1}{K(P)}=-\frac{1}{V}\left(\frac{\partial V}{\partial P}\right)_{T}=c_{0}+c_{1} P+c_{2} P^{2} \cdots+c_{n} P^{n}+\cdots
$$

Under stretching $(P<0)$, the true ground state is fractured into pieces (relieving the strain energy). As a result, $P=0$ can not be a point of analyticity for $K(P)$, and thus (1) has zero radius of convergence.

Similar arguments were used by Dyson [1] in 1952, where he argued that calculations in quantum electrodynamics, expressed as a power series in the fine structure constant $e^{2} / \hbar c \approx 1 / 137$, had zero radius of convergence (because negative values of $e^{2}$ lead to unstable theories). This did not prevent these calculations from being useful (indeed, they represent the best quantitative agreement between theory and experiment known to science). The community believes these expansions are asymptotic in the same sense as Stirling's approximation $\Gamma(n)=(n-1) ! \sim \mathrm{e}^{-\mathrm{n}} \mathrm{n}^{\mathrm{n}}(2 \pi / \mathrm{n})^{1 / 2}\left(1+1 /(12 \mathrm{n})+1 /\left(288 \mathrm{n}^{2}\right)+\cdots\right)$ : at any fixed $n$ no matter how large, Stirling's series in $1 / n$ eventually diverges, but the difference between the function and the $M^{\text {th }}$ approximation goes to zero faster than $1 / n^{M}$ as $n \rightarrow \infty$.

Since Dyson's work, field theoretic methods have been developed [2] 5 ] to relate the instabilities in the theories at small negative couplings to the high-order terms in perturbation theory. Here we apply these methods to a particular case, using the statistical mechanics of thermally nucleated cracks to calculate the high-order terms $c_{n}$ in the inverse bulk modulus (1). Statistical and thermodynamic approaches to crack nucleation and fracture have an established history [6]. However, most work in this area is concentrated on failure at rather high stresses, near the threshold for instability (the spinodal point). The high-order terms in the perturbative expansion of the inverse bulk modulus are governed by the elastic response of the material to infinitesimally small tension (see below), so we are far away from the spinodal point and linear elastic theory is an adequate description.

Consider an infinite two-dimensional isotropic linear-elastic material subject to uniform compression $P$ at infinity. Creation of a cut of length $\ell$ will increase the energy by $2 \alpha \ell$, where $\alpha$ is the surface tension (the energy per unit length of edge), with a factor of 2 because of the two free surfaces. On the other hand, for negative $P$ (uniform tension) the cut will open up because of elastic relaxation. Calculating this relaxation energy we find the total energy $E$ of a crack of length $\ell$ :

$$
E(\ell)=2 \alpha \ell-\frac{\pi P^{2}\left(1-\sigma^{2}\right) \ell^{2}}{4 Y}
$$

Introducing

$$
\ell_{c}=\frac{4 Y \alpha}{\pi P^{2}\left(1-\sigma^{2}\right)}
$$


we can rewrite the energy of the crack as

$$
E=2 \alpha \ell-\alpha \frac{\ell^{2}}{\ell_{c}}
$$

It follows that cracks with $\ell>\ell_{c}$ will grow, giving rise to the fracture of the material, while those with $\ell<\ell_{c}$ will heal - a result first obtained by Griffith [7. This is the instability that is responsible for the breakdown of elastic perturbation theory. Because the energy $E\left(\ell_{c}\right)=\alpha \ell_{c}$ grows as $1 / P^{2}$ as $P \rightarrow 0$, interactions between thermally nucleated cracks are unimportant at small $P$ and low temperatures (allowing us to use the "dilute gas approximation").

The thermodynamic properties of a macroscopic system can be obtained from its partition function $Z$ :

$$
Z=\sum_{n} \exp \left(-\beta E_{n}\right)
$$

where the sum is over all states of the system. Once a perturbative expansion for the free energy $F=-(1 / \beta) \ln Z$ is known, one can calculate the power series expansion for the inverse bulk modulus using

$$
\frac{1}{K(P)}=-\frac{1}{P A}\left(\frac{\partial F}{\partial P}\right)_{T}
$$

where the elastic material has area $A$.

For $P<0$, our model is in a metastable state, and direct computation of the partition function should yield a divergent result. A similar problem for the three-dimensional Ising model was solved by Langer [8]: one has to compute the partition function in a stable state $P>0$, and then do an analytical continuation in parameter space to the state of interest. The free energy develops an imaginary part in the unstable state, related to the decay rate for fracture [9]: the situation is similar to that of barrier tunneling in quantum mechanics [10], where the imaginary part in the energy gives the decay rate of a resonance.

The calculation of the imaginary part of the partition function is dominated by a saddle point, that in our case is a straight cut of length $\ell_{c}$. The straight cut is the saddle point because it gains the most elastic relaxation energy for a given number of broken bonds (as can be checked with a direct calculation [1] ).

For simplicity, we start by considering the model without including the quadratic fluctuations around the saddle point. The partition function for a dilute gas of straight cuts of arbitrary length at their equilibrium shape for the tension $P$ can be calculated directly: the imaginary part of the free energy for $P<0$ is

$$
\operatorname{Im} F^{\text {simple }}(P)=\frac{2}{\beta|P|}\left(\frac{Y}{\beta \lambda^{2}\left(1-\sigma^{2}\right)}\right)^{1 / 2}\left(2 \pi \frac{A}{\lambda^{2}}\right) \exp \left\{\frac{-4 \beta Y \alpha^{2}}{\pi P^{2}\left(1-\sigma^{2}\right)}\right\}
$$

with $\lambda$ being the ultraviolet cutoff in the theory (roughly, the interatomic distance). (The factor $\left(2 \pi A / \lambda^{2}\right)$ comes from the zero modes for rotating and translating the cracks.)

Assuming the free energy is analytic in the complex $P$ plane except for a branch cut along $P \in(-\infty, 0]$, we obtain a Cauchy representation [3,5] for the free energy

$$
F(P)=\frac{1}{\pi} \int_{-\infty}^{0} \frac{\operatorname{Im} F\left(P^{\prime}\right)}{P^{\prime}-P} d P^{\prime}
$$

As was first established for similar problems in field theory [2 [4], this relation determines the high-order terms in perturbative expansion of the free energy $F(P)=\sum_{n} f_{n} P^{n}$

$$
f_{n}=\frac{1}{\pi} \int_{-\infty}^{0} \frac{\operatorname{Im} F\left(P^{\prime}\right)}{P^{\prime n+1}} d P^{\prime}
$$

Because the saddle point calculation becomes more and more accurate as $P \rightarrow 0$, and because the integrals in equation (9) are dominated by small $P$ as $n \rightarrow \infty$, using the saddle-point form for the imaginary part of the free energy yields the correct $n \rightarrow \infty$ asymptotic behavior of the high-order coefficients $f_{n}$ in the free energy. For our initial approximation (7) 


$$
f_{n}^{\text {simple }}=(-1)^{n+1} \Gamma\left(\frac{n+1}{2}\right)\left(\frac{\pi\left(1-\sigma^{2}\right)}{4 \beta Y \alpha^{2}}\right)^{n / 2}\left(2 \pi \frac{A}{\lambda^{2}}\right) \frac{1}{2 \pi^{1 / 2} \beta^{2} \alpha \lambda}
$$

We can then use the thermodynamic relation (6) to show $c_{n}=-(n+2) f_{n+2} / A$, and thus calculate the asymptotic behavior of the expansion of the bulk modulus:

$$
\frac{c_{n+1}}{c_{n}} \rightarrow-n^{1 / 2}\left(\frac{\pi\left(1-\sigma^{2}\right)}{8 \beta Y \alpha^{2}}\right)^{1 / 2} \quad \text { as } \mathrm{n} \rightarrow \infty
$$

which indicates that the high-order terms $c_{n}$ in the perturbation expansion for the inverse bulk modulus roughly grow as $(n / 2)$ !. The formula (11) will still be correct once we add the quadratic fluctuations (although $\alpha$ will develop temperature-dependent corrections). For cracks in three dimensions, we expect the scaling $c_{n+1} / c_{n} \sim n^{1 / 4}$ as $n \rightarrow \infty$, using similar arguments.

The above calculation ignores the quadratic fluctuations about the saddle point, which will change the prefactor in the expression (7) for the imaginary part of the free energy and will renormalize the surface tension $\alpha$. There are two kinds of quadratic fluctuations we have to deal with. (I) Curvy cuts - changes in the shape of the tear in the material: deviations of the broken bonds from a straight-line configuration. (II) Surface phonons - thermal fluctuations of the free surface of the crack about its equilibrium opening shape. Since the energy of the curvy cracks is calculated for their equilibrium configuration, the response of the surface phonons to curving the cut is already incorporated, so the quadratic fluctuations (II) can be calculated independently from (I).

In both cases the theory needs regularization: we must decide exactly how to introduce the ultraviolet cut-off $\lambda$. We describe the curvy crack as a bunch of line segments, parameterized by kink angles $\alpha_{i}-$ angles between consecutive segments. We calculate the energy release in the material with a crack using a conformal mapping from the plane with a cut to the exterior of a unit circle [12], which for a piecewise linear cut is given by a modified Schwartz-Christoffel map. It is convenient for the regularization of the surface phonons to use equally spaced points on the unit circle, of angular spacing $\pi \lambda / \ell$; this corresponds to points which accumulate at the edges of the original crack cut. For consistency, we use the same regularization for the curvy crack segments. We have also used the (less convenient, more natural) regularization of equally spaced points along the original crack cut, and the results are similar (although the convergence is not as convincing): the form of the imaginary part of the free energy and the ratio of the high-order elastic coefficients (11) do not change, but (see below) the constants $s_{0}, s_{1}$ and $s_{2}$ and the temperature-dependence of $\alpha$ do change [11].

To calculate the quadratic fluctuations due to the surface phonons, we must calculate the determinant of the matrix $M_{p}$, given by the energy spectrum of the normal modes about the crack's equlibrium (saddle point) opening:

$$
\operatorname{det} M_{p}=2 \pi\left(\frac{2\left(1-\sigma^{2}\right)}{\beta Y \lambda^{2}}\right)^{2} n \exp \left\{n\left(2 \ln \frac{\beta Y \lambda^{2}}{2\left(1-\sigma^{2}\right)}-2\right)\right\}
$$

where $n$ is number of kinks. Similarly, the quadratic fluctuations due to curving the crack is given by the determinant of the matrix $M_{i j}$ that specifies the change in energy release to quadratic order in the kink angles $\alpha_{i}$ (so the energy change due to the curving of a straight cut $\Delta E\left(\left\{\alpha_{i}\right\}\right)=M_{i j} \alpha_{i} \alpha_{j}+O\left(\alpha^{3}\right)$ ). We have obtained an analytical expression for the entries of the above matrix by calculating, to quadratic order, the energy of a crack with two kinks. This lengthly and tedious calculation [11] was checked both using a finite-element crack simulation [13] and an exact analytical solution for a cut that is an arc of a circle. We assume that scaling of the curvy crack determinant is of the same form as for the surface phonons :

$$
\operatorname{det} M \sim s_{0} n^{s_{1}} \exp \left\{n\left(\ln \frac{\beta \alpha \lambda}{4 \pi^{3}}+s_{2}\right)\right\}
$$

as the number of kinks $n \rightarrow \infty$. We extract the constants $s_{0}, s_{1}$, and $s_{2}$ numerically, using systems with up to $n=400$ kinks: we extrapolate visually to find $\ln s_{0}=1000 \pm 1000, s_{1}=-400 \pm 100$, and $s_{2}=7.5 \pm 0.5$. Notice that $s_{0}$ is subdominant to $s_{1}$, which is subdominant to $s_{2}$, so our errors get rather large - even without including our uncertainty as to the scaling form (13).

These quadratic fluctuations modify the imaginary part of the free energy (7) as follows:

$$
\begin{aligned}
\operatorname{Im} F(P)= & \frac{s_{0}}{\sqrt{8 \pi}} \frac{\beta Y \lambda^{2}}{1-\sigma^{2}}\left(\frac{l_{c}}{\lambda}\right)^{-\left(1+s_{1}\right) / 2} \\
& \exp \left\{\frac{l_{c}}{\lambda}\left(1-\frac{s_{2}}{2}+\ln \frac{2\left(1-\sigma^{2}\right)}{\beta Y \lambda^{2}}+\frac{1}{2} \ln \frac{4 \pi^{3}}{\beta \alpha \lambda}\right)\right\} \operatorname{Im} F^{\text {simple }}(P)
\end{aligned}
$$


with $l_{c}$ given by (3) and $\operatorname{Im} F^{\text {simple }}(P)$ given by (7). The first term in (14) modifies the prefactor, the other one effectively renormalizes the surface tension:

$$
\alpha_{r}=\alpha+\frac{1}{2 \beta \lambda}\left(\frac{s_{2}}{2}-1+\ln \frac{\beta Y \lambda^{2}}{2\left(1-\sigma^{2}\right)}+\frac{1}{2} \ln \frac{\beta \alpha \lambda}{4 \pi^{3}}\right)
$$

Finally, we derive the correct asymptotic form for $c_{n}$ :

$$
c_{n}=(-1)^{n}(n+2) \Gamma\left(\frac{n-s_{1}}{2}\right)\left(\frac{\pi\left(1-\sigma^{2}\right)}{4 \beta Y \alpha_{r}^{2}}\right)^{n / 2}\left(\frac{2 \pi}{\lambda^{2}}\right)\left(\frac{\beta \alpha_{r}^{2} \lambda}{\alpha}\right)^{s_{1} / 2} \frac{s_{0} Y \lambda}{8 \sqrt{2 \pi} \beta \alpha\left(1-\sigma^{2}\right)}
$$

This is the main result of our paper.

We warn the reader to treat our results in the proper context. First, we do not expect this calculation to have experimental implications in the near future. Real fracture invariably occurs on inhomogeneities in the material: pre-existing surface or bulk microcracks, dislocation tangles, or grain boundaries. Even for a perfect dislocation-free crystal with stabilized surfaces, the effects we describe will remain immensely small: the reason that incredibly tiny compression make (relatively) large changes in volume (leading to large high-order terms in the power series) is because they suppress the (already incredibly rare) opening of large thermally nucleated cracks: measuring these effects would seem infeasible.

Second, our results can be viewed as a straightforward extension to the solid-gas sublimation point of Langer [8,9] and Fisher's [14] theory of the essential singularities at the liquid-gas transition. Indeed, if we allow for vapor pressure in our model, then our system will be in the gas phase at $P=0$. The essential singularity we calculate shifts from $P=0$ to the vapor pressure. If we measure the nonlinear bulk modulus as an expansion about (say) atmospheric pressure, it could converge: but the radius of convergence would be bounded by the difference between the point of expansion and the vapor pressure.

Third, we have forbidden dislocation nucleation and plastic flow in our model. Dislocation emission is crucial for ductile fracture, but by restricting ourselves to a brittle fracture of defect-free materials we have escaped many (lethal) complications. Dislocations are in principle important: the nucleation 15] barrier $E_{d i s}$ for two edge dislocations in an isotropic linear-elastic material under uniform tension $P$ with equal and opposite Burger's vectors $\vec{b}$ is

$$
E_{d i s}=\frac{Y b^{2}}{4 \pi\left(1-\sigma^{2}\right)} \ln \frac{Y}{P}+E_{0}
$$

where $E_{0}$ is a $P$ independent part that includes the dislocation core energy. The fact that $E_{\text {dis }}$ grows like $1 / \ln P$ as $P \rightarrow 0$ (much more slowly than the corresponding barrier for cracks) tells that in more realistic models dislocations and the resulting plastic flow [16] cannot be ignored. While dislocations may not themselves lead to a catastrophic instability in the theory (and thus to an imaginary part in the free energy?), they will strongly affect the dynamics of crack nucleation as noted above.

Fourth, we are being careful to distinguish tension from negative hydrostatic pressure. The response of a crystal to a force depends in a fundamental way on whether the force couples to the atoms or to the lattice (i.e., to the broken symmetry). Under gravity, all crystals will flow like anisotropic liquids, with a viscosity given simply in terms of the vacancy diffusion constant. In contrast, a crystal gripped at the sides and strained will not flow with a rate linear in the external force: under a strain produced by coupling to the surface layers, single vacancies cannot individually relieve the strain, and a dislocation pair (a loop in a three-dimensional case) must appear in the material for strain relief to occur: the plastic response of a two-dimensional crystal to external strain is a temperature-dependent powerlaw in the external force [16]. Although we call the compression (or tension) $P$, it is not hydrostatic pressure, but a coupling to the lattice. Under negative hydrostatic pressure, vacancies will have a negative chemical potential $\mu$, and the dominant fracture mechanism becomes the nucleation of vacancy clusters or voids (rather than Griffith-type critical microcracks), as noted by Golubović and collaborators [17]. For negative chemical potential $\mu$ per unit area, their vacancy clusters (up to constants) have energy $E_{v a c}(R) \sim \alpha R-|\mu| R^{2}$. If we identify $\mu$ with $P$, comparing with (2) we see that the vacancy cluster gains an energy linear in $P$, while the crack gain is quadratic. This leads to a problem that maps onto Langer's calculation, leading to the asymptotic relation $c_{n+1} / c_{n} \sim n$, so the coefficients in this case would diverge more strongly: $c_{n} \sim n$ !. However, the identification of $\mu$ with $P$ demands a mechanism for relieving elastic tension by the creation of vacancies. Ignoring possible effects of the boundaries (presumed infinitely far removed), as noted above vacancies will be created (and hence relieve tension) only through dislocation motion, which we have explicitly excluded from our model. 
We acknowledge the support of DOE Grant DE-FG02-88-ER45364. We would like to thank Yakov Kanter, Eugene Kolomeisky, Paul Houle, Tony Ingraffea, Paul Wawrzynek, and Robb Thompson for useful conversations.

[1] F. J. Dyson, Phys. Rev. 85, 631 (1952).

[2] C. M. Bender and T. T. Wu, Phys. Rev. 184, 1231 (1969).

[3] E. Breźin, J. C. Le Guillou and J. Zinn-Justin, Phys. Rev. D 151558 (1977).

[4] G. Parisi, Phys. Lett. B 66167 (1977).

[5] E. B. Bogomolny, Phys. Lett. B 67193 (1977).

[6] R. I. B. Selinger, Z. Wang and W. M. Gelbart, Phys. Rev. A 434396 (1990) and references therein.

[7] A. A. Griffith, Philos. Trans. Roy. Soc. London Ser. A 221163 (1920).

[8] J. S. Langer, Ann. Phys. 41108 (1967); see also N. J. Günther, D. A. Nicole and D. J. Wallace, J. Phys. A 13 1755 (1980).

[9] J. S. Langer, Ann. Phys. 54258 (1969).

[10] I. Affleck, Phys. Rev. Lett. 46388 (1981).

[11] A. Buchel and J. P. Sethna (manuscript in preparation).

[12] N. I. Muskhelishvili, "Some basic problems of the mathematical theory of elasticity; fundamental equations, plane theory of elasticity, torsion and bending", Groningen, P. Noordhoff (1963).

[13] FRANC2D, P. A. Wawrzynek and A. R. Ingraffea, Cornell Fracture Group. More information is available at http://pilsner.cfg.cornell.edu.

[14] M. E. Fisher, Physics 3255 (1967).

[15] D. R. Nelson, Phys. Rev. B 182318 (1978).

[16] Two-dimensional dislocation-mediated plastic flow is closely related to the problem of vortex nucleation and superflow decay in superfluid films: V. Ambegaokar, B. I. Halperin, D. R. Nelson, and E. D. Siggia, Phys. Rev. Lett. 40, 783 (1978); V. Ambegaokar, B. I. Halperin, D. R. Nelson, and E. D. Siggia, Phys. Rev. B 21, 1806 (1980); P. Minnhagen, O. Wetman, A. Jonsson, and P. Olsson, it Phys. Rev. Lett. 74, 3672 (1995).

[17] L. Golubović and A. Peredera, Phys. Rev. E 512799 (1995). They conclude with the interesting and apparently correct statement that voids smaller than the Griffith length can nonetheless grow and fracture the material. 